\title{
Micro-fabrication of advanced photonic devices by means of direct point-by-point femtosecond inscription in silica
}

\author{
Vladimir Mezentsev, Mykhaylo Dubov, Amos Martinez, Yicheng Lai, Thomas P. Allsop, \\ Igor Khrushchev, David J. Webb, Filip Floreani, and Ian Bennion \\ Photonics Research Group, Aston University, Birmingham B4 7ET, United Kingdom
}

\begin{abstract}
We present recent results on experimental micro-fabrication and numerical modeling of advanced photonic devices by means of direct writing by femtosecond laser. Transverse inscription geometry was routinely used to inscribe and modify photonic devices based on waveguiding structures. Typically, standard commercially available fibers were used as a template with a pre-fabricated waveguide. Using a direct, point-by-point inscription by infrared femtosecond laser, a range of fiber-based photonic devices was fabricated including Fiber Bragg Gratings (FBG) and Long Period Gratings (LPG). Waveguides with a core of a couple of microns, periodic structures, and couplers have been also fabricated in planar geometry using the same method.
\end{abstract}

Keywords: Femtosecond inscription, microfabrication, fiber sensors

\section{INTRODUCTION}

In recent years, the technique of laser inscription in transparent dielectrics by means of focused femtosecond laser irradiation has attracted considerable interest. The method has been applied to bulk glass, optical fibers, crystals, and polycrystalline or ceramic media. The method appears to be a promising and flexible technology for optical device fabrication. In particular, fabrication of fiber gratings has been demonstrated, including directly written long-period gratings, ${ }^{1,2}$ fiber Bragg gratings (FBGs) produced using special phase-masks, ${ }^{3,4}$ and FBGs produced by a femtosecond UV laser using standard phase-masks. ${ }^{5}$ Recently, a method was reported for direct writing of FBGs using point-by-point irradiation by an infrared femtosecond laser. ${ }^{6}$ This method requires neither phase masks nor photosensitized fibers and hence offers a remarkable technological flexibility with a very short processing time.

\section{EXPERIMENTAL SETUP}

The schematic of the experimental set-up is presented in Figure 1. Pulse source comprises the laser system (Spectra Physics), the Spitfire amplifier (Positive Light), and Tsunami femtosecond mode-locked generator (Spectra Physics). It is capable to produce 120-150 fs pulses at the wavelength of $796 \mathrm{~nm}$ at $1 \mathrm{kHz}$ repetition rate. The computer controlled attenuation is realized with the half wave-plate (2) mounted on the motorized rotational stage, and a Glan prism (3). Electronic shutter (1) is installed immediately after the laser system output window. He-Ne laser (not shown in the Figure) is used for alignment. The optical fiber (6) is mounted on the computer controlled 2D-stage (7) (ABL1000, Aerotech) and moved with a speed of 0.07-5 mm per second. The femtosecond (fs) inscription of the grating or other structure is performed after careful positioning of the focal spot with respect to the fiber core on both ends of the scanning range with the two auxiliary 3D-stages (5). Typically, $\times 100$ long working distance objective (ApoSL, Mitutoyo) was used to focus the laser radiation. Two CCD-cameras (8) with optical zooming systems were used for alignment and on-line monitoring of the inscription process.

Fine tuning of extremely stable laser setup is required to achieve pinpoint accuracy of the focused femtosecond pulses which allows for robust reproducible sub-micron fabrication of virtually arbitrary geometric structures with a distributed variation of refractive index.

Further author information: (Send correspondence to V.M.)

V.M.: E-mail: v.mezentsev@aston.ac.uk, Telephone: +44 1212043481

Laser-based Micropackaging, edited by Friedrich G. Bachmann, Willem Hoving, Yongfeng Lu, Kunihiko Washio, Proc. of SPIE Vol. 6107, 61070C, (2006) · 0277-786X/06/\$15 - doi: 10.1117/12.647249 


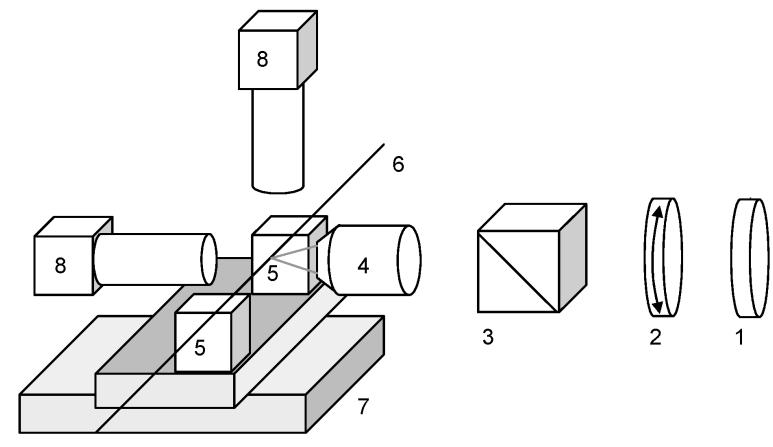

Figure 1. Optical layout for femtosecond fiber grating inscription. The light passes a shutter (1), a half-wave plate (2), Glan prism (3), x100 long working distance microscopic objective (4), and is focused inside the fiber (6). Two alignment 3D-stages (5) are mounted on top of the high precision computer controlled 2D-stage (7).

\section{DIRECT FS-INSCRIPTION OF FIBER BRAGG GRATINGS}

In this Section, we present a method for inscribing FBGs using direct, point-by-point writing by an infrared femtosecond laser. This method requires neither phase-masks nor photosensitized fibers and hence offers remarkable technological flexibility. Unlike known point-by-point methods of making $\mathrm{FBGs}^{7}$ it requires a very short inscription time of less than a minute per grating. High quality, strongly-reflecting gratings were produced using this method in commercially available, non-photosensitized, unhydrogenated fibers.

The experimental setup is shown in Fig. 1. The beam is focused into the fiber core by either a $\times 40$ or a $\times 100$ microscopic objective. Pulse energy was varied from 0.3 to $1 \mathrm{~mJ}$, corresponding to peak intensities in the order of $10^{14} \mathrm{~W} / \mathrm{cm}^{2}$. The grating length ranged between 10 and $55 \mathrm{~mm}$. Standard telecommunication fiber (SMF) and dispersion shifted fiber (DSF) were used; no photosensitization procedure was carried out prior to the exposure. The fiber was placed on a high-precision, two coordinate translation stage. The stage moved at a constant speed along the fiber axis, translating the fiber with respect to the focal point of the beam. Each laser pulse produced a grating pitch in the fiber core. Therefore, the grating period was set by changing the ratio of the translation speed to the pulse repetition rate. Gratings of first, second and third order were inscribed at speeds of $0.535,1.07$ and $1.605 \mathrm{~mm} / \mathrm{s}$, respectively.

Reflection and transmission spectra were monitored in situ using two optical spectrum analyzers ${ }^{6}$ (not shown in Fig. 1). After the inscription, the gratings were characterized using a tunable laser with a linewidth of $1 \mathrm{pm}$ and a high-performance analyzer with a resolution of $5 \mathrm{pm}$.

Transmission spectra of first-, second- and third-order FBGs are presented for comparison in Fig. 2. The three gratings have been written in segments of DSF using a $\times 100$ microscope objective. In this experiment, the second-order grating shows the strongest response. This is in line with an earlier study of gratings produced by femtosecond writing with a phase mask. ${ }^{3}$ The increased efficiency of higher orders is a feature of the femtosecond inscription process, which differs from the conventional UV inscription when sinusoidal modulation of refractive index is usually achieved. This feature is due to the very localized inscription domain, created by a tightly focused laser beam. Hence, the modulation of refractive index in fs-inscribed structures is intrinsically unharmonic which results in higher order gratings being more efficient. Localization of refractive index modulation is further emphasized by the nonlinear nature of the process. The physical mechanism of femtosecond inscription involves nonlinear absorption and multi-photon ionization. Consequently, the shape of the inscribed features does not reproduce linearly the intensity profile of the laser beam. For example, features as small as $0.3 \mu \mathrm{m}$ can be produced at a wavelength of $800 \mathrm{~nm}$, which is well beyond the resolution imposed by diffraction limit. ${ }^{8}$ There is also evidence that the refractive index modification produced by femtosecond writing is significantly larger than that produced by UV writing. ${ }^{2}$ As a result of the above, the profile of refractive index modulation in a fs-inscribed grating along the fiber axis is likely to consist of sharp features. Hence, higher-order components

Proc. of SPIE Vol. 6107 61070C-2 


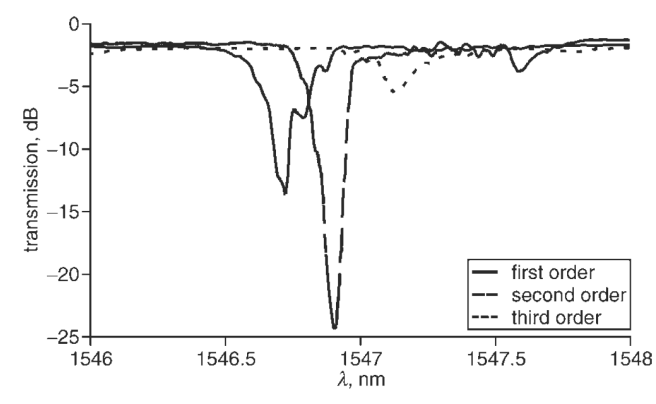

a

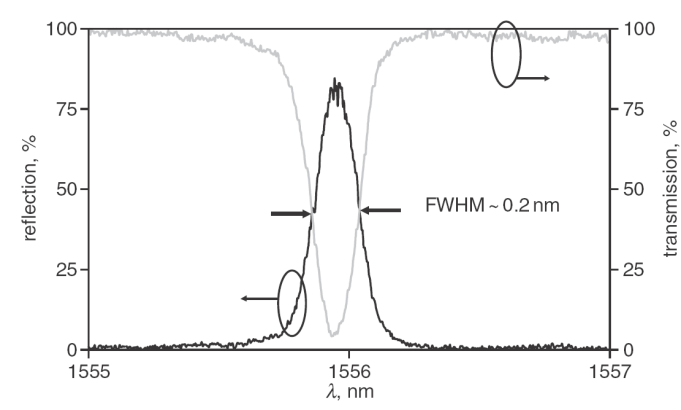

$\mathrm{b}$

Figure 2. Measured spectra: (a) transmission spectra in gratings of first, second and third orders; (b) reflection and transmission spectra of second-order grating.

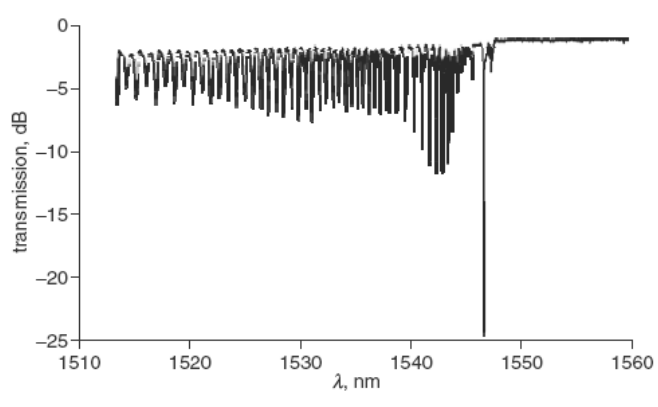

a

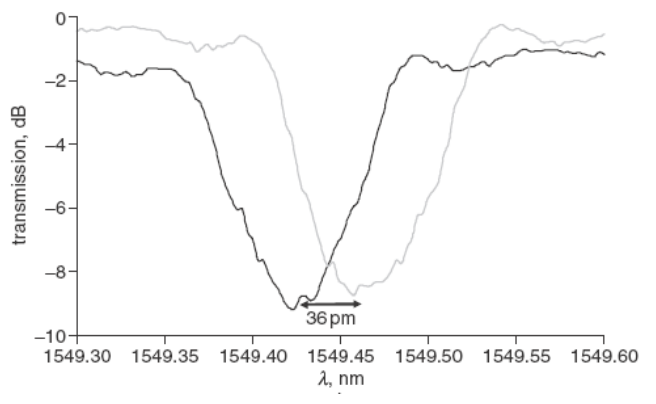

$\mathrm{b}$

Figure 3. Grating spectrum showing strong coupling to cladding modes, and transmission spectra measured in two orthogonal polarizations, corresponding to refractive index anisotropy of $3.6 \times 10^{-5}$

(a) Grating spectrum (b) Transmission spectra : solid line — fast axis, gray line — slow axis.

in the spectra of fs-inscribed FBGs are more pronounced than those in the UV-written devices. However, the relatively large size of the laser spot in the fiber core causes overlapping of pitches in the gratings of lower orders, thus reducing their quality. For example, the first-order resonance did not appear at all in the structures produced using a $\times 40$ objective with a focal spot larger than that of a $\times 100$ objective. The FWHM linewidth of the grating resonance typically ranged between 0.1 and $0.2 \mathrm{~nm}$ and did not show a distinct dependence on the grating order. Typical transmission and reflection spectra are given in Fig. 2b. This second-order grating was inscribed in SMF using a $\times 100$ objective. Non-resonant (out of band) losses were typically less than $1.5 \mathrm{~dB}$.

A significant coupling to the cladding modes was observed in a broad spectral window spreading towards short wavelengths in all the gratings, as illustrated by Fig. 3a. Cladding mode coupling is noticeable as far as 50 $\mathrm{nm}$ away from the resonant frequency. Another characteristic of these gratings was a considerable polarization dependence. The spectral separation between orthogonal polarizations was typically in the range 20-36 pm, corresponding to the birefringence as high as $32 \times 10^{-6}$, which is much higher than that in the UV inscribed gratings.

\section{VECTOR BENDING SENSOR BASED ON FS-INSCRIBED FBG}

Direction-sensitive, or vectorial, fiber grating sensors have been a subject of intense research for many years. Usually, fiber grating-based vector sensors can only be realized in specially designed fibers, such as multicore fiber, ${ }^{9}$ D-shaped fiber or flat-clad fiber. ${ }^{10}$ In all these devices, the direction-selective sensitivity is based on the broken cylindrical symmetry of the fiber. High manufacturing cost of specialist fibers and the need for dedicated coupling and interrogation techniques considerably reduce the practicality of this type of sensors. Fabrication of vector sensors in fibers of conventional, circular, geometry would be a very attractive proposition as this 

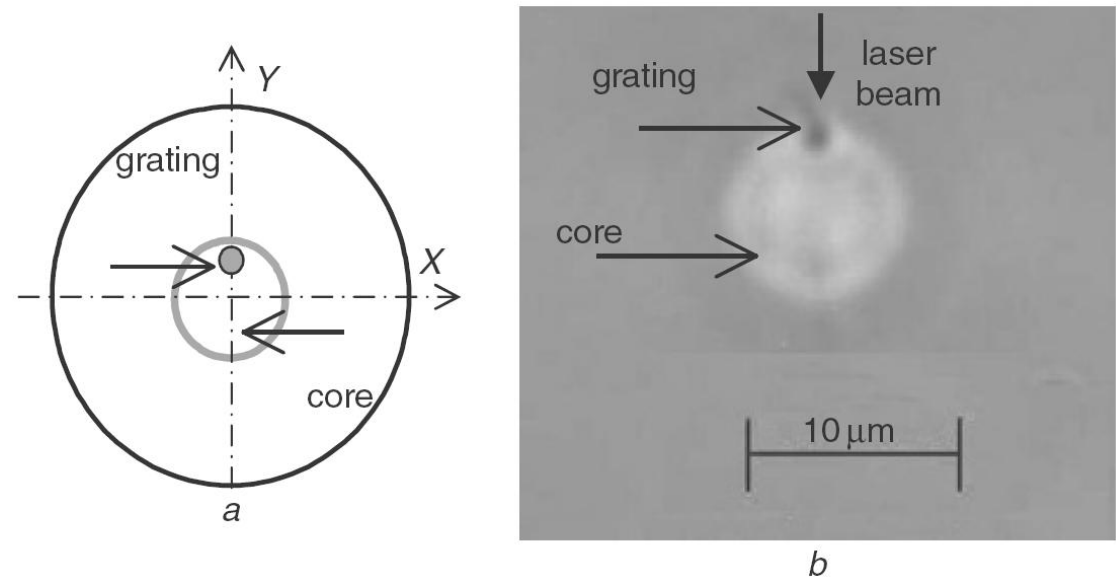

Figure 4. Schematic of cross-sectional profile of fs-inscribed FBG, and microscopic image of its cross-section. Grating is inscribed off centre to create required asymmetry in device and thus directional bending sensitivity. (a) Schematic (b) Microscopic image.

would alleviate the need for special fibers and reduce the complexity and cost of the interrogation systems. A straightforward way to achieve directional sensitivity would be of course to introduce the required asymmetry by the inscription process itself. However, this is difficult to realize using conventional inscription techniques, based on exposure of fiber to ultraviolet radiation transmitted through a mask, as these usually affect a large fraction of the fiber cross-section and generally do not allow to control precisely the transverse structure of inscribed features. Precise positioning and high-contrast of introduced modifications in the fiber material can be achieved by employing a novel technique of femtosecond laser inscription. In this section, we demonstrate a novel, direction-sensitive bending sensor based on an asymmetric fiber Bragg grating (FBG) inscribed by an infrared femtosecond laser. The technique is based on tight transverse confinement of the fs-inscribed structures and can be directly applied in conventional, untreated singlemode fibers.

Fabrication of FBGs by focused radiation of a femtosecond laser has been reported recently by several groups. Inscription by a UV femtosecond laser ${ }^{5}$ and an infrared femtosecond laser with a specially designed phase mask ${ }^{3}$ has been demonstrated. We have recently presented the fabrication of FBGs using a direct, point-by-point writing technique $^{6}$ that allows the inscription of high quality, strong FBGs in untreated standard fibers. Fs-inscribed gratings present a number of interesting characteristics, such as high thermal stability, ${ }^{3,11}$ large refractive index modulation and, notably, confinement of induced modification of material within a fraction of the fiber cross-section with an area much smaller than the core area. ${ }^{6}$ fiber grating structures can be precisely placed in any desired location within or in the vicinity of the core. The transverse size and shape of the structure can be controlled by the focusing conditions and the exposure regime. By inscribing an axially-offset FBG, an intrinsically asymmetric FBG device can be produced. When acting as a bending sensor, the device shows a shift of the Bragg resonance which depends on the orientation of the grating structure with respect to the bending direction.

The laser beam was attenuated by a variable attenuator and subsequently focused into the fiber core by a $\times 100$ microscopic objective. The pulse energy after the objective was approximately $0.5 \mathrm{~mJ}$. Standard singlemode fiber was used without any preparation or photosensitization carried out prior to the exposure. The fiber was placed on a high-precision, two-coordinate translation stage and aligned in such way that the beam was focused in a plane located $3 \mu \mathrm{m}$ before the fiber axis. The length of the fabricated grating was $40 \mathrm{~mm}$. Figure 4 shows the cross-section of the inscribed grating. The grating cross-section was elongated along the direction of the laser beam with the transverse dimensions of approximately 1 by $2 \mu \mathrm{m}$. The grating is clearly offset from the centre, inducing the required asymmetry in the device and therefore inducing directional bending sensitivity. The grating was then tested in a bending rig, as illustrated in Fig. 5. The grating spectra were measured using a tunable laser in conjunction with an optical spectrum analyzer (OSA). The system resolution was as good as 


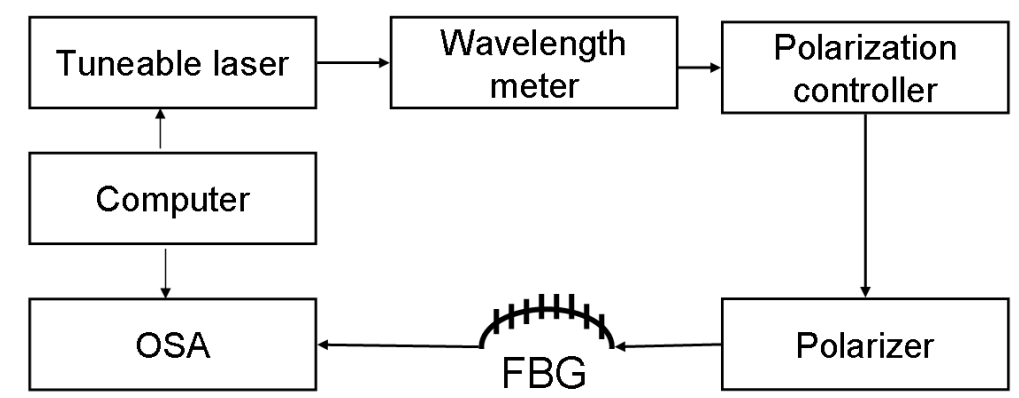

Figure 5. Bending measurement setup PC: polarisation controller; OSA: optical spectrum analyser

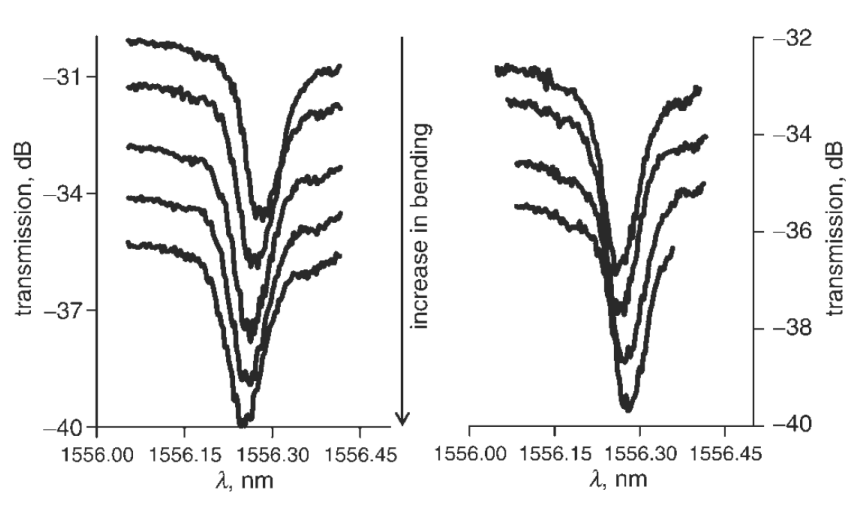

a

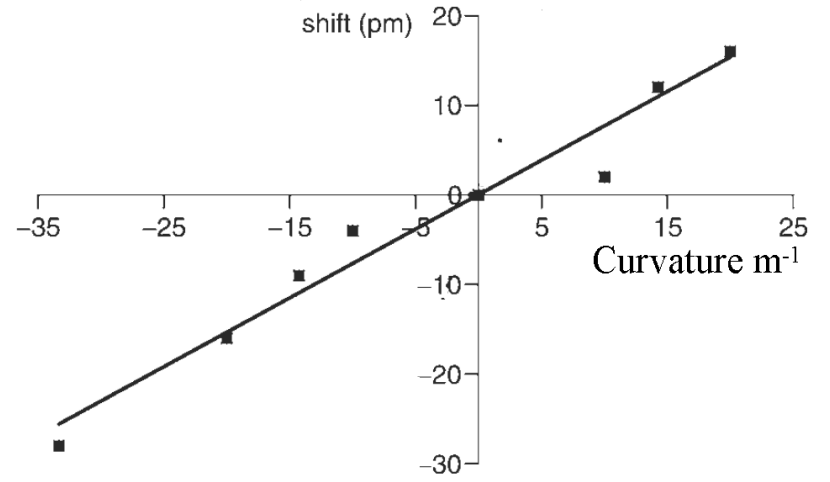

$\mathrm{b}$

Figure 6. Spectral profiles showing blue and red Bragg wavelength shifts depending on bending direction, and wavelength shift against bending strength and direction a Spectral profiles b Wavelength shift against bending strength and direction.

$1 \mathrm{pm}$. As the fs-inscribed gratings typically exhibit strong polarization dependence, special measures were taken to ensure that all measurements be carried out along the same polarization axis. The variations of the Bragg wavelength caused by concave and convex bends are shown in Fig. 6. Blue and red spectral shifts, corresponding to the opposite bending directions can be clearly seen, indicating the directional sensitivity of the device.

One can suggest that the sensitivity of the device can potentially be improved by inscribing smaller spatial features and perhaps by implementing more complex grating designs aimed at maximizing the effect of strain. It is also important that the demonstrated device is designed for detection of bending only in the plane fiber axisBragg grating. Independent sensitivity in the orthogonal plane could be attained by inscribing another, spectrally-shifted grating structure, in a different sector of the fiber cross-section.

\section{FS-INSCRIBED LONG PERIOD GRATINGS}

\subsection{LPG-based directional bend sensor}

A long period grating (LPG) is an axially periodic refractive index variation in the core of a single-mode optical fiber that couples light in the core to fiber-cladding modes at discrete wavelengths ${ }^{12}$; LPGs have been suggested for many potential applications in the field of sensing. ${ }^{13}$ A promising application of fiber LPG devices is as a curvature sensor, ${ }^{14}$ but a problem here is that the direction of the bend cannot generally be determined and thus at present LPGs written in standard fiber cannot be used as true orientation sensors. Some limited success in making orientation sensors has been achieved with LPGs written into asymmetric fibers such as D-shaped cladding fiber ${ }^{15}$ or eccentric core fiber. ${ }^{16}$ Although the resulting devices can determine the orientation of the bend, they still present some practical problems; for example, these fibers are generally not easy to connect to standard optical fiber, thus adding complexity and cost to the fabrication procedure for sensor arrays. In this 


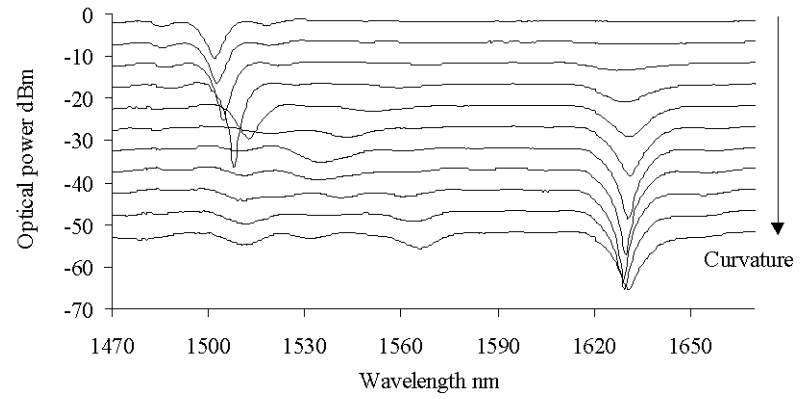

a

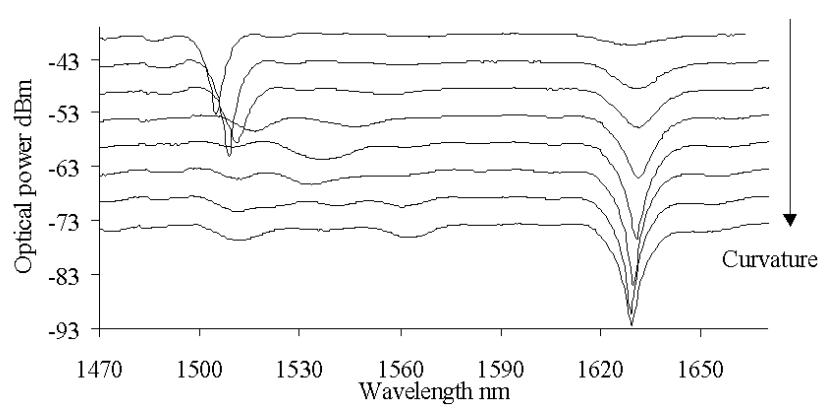

$\mathrm{b}$

Figure 7. Transmission spectrum of LPG fiber device against direction and amount of curvature: (a) concave (b) convex

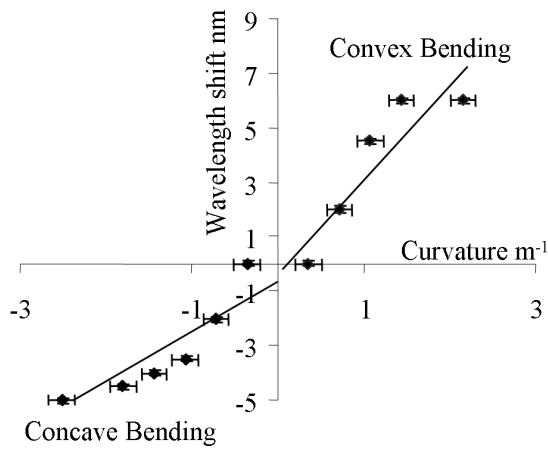

a

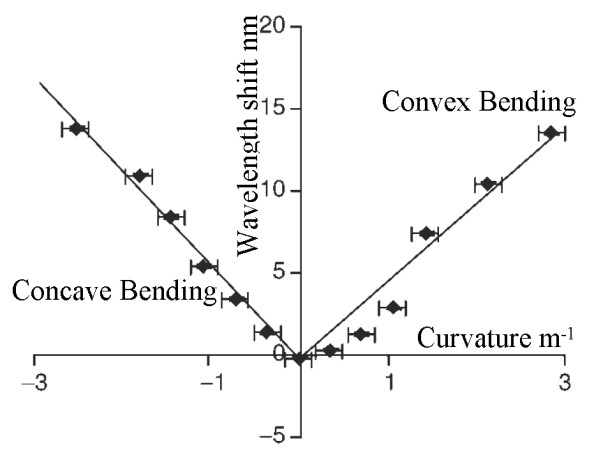

$\mathrm{b}$

Figure 8. Spectral sensitivity of induced attenuation bands (a) and normal attenuation band (b) at $1500 \mathrm{~nm}$

Section describe the fabrication and characterization of an LPG sensor in standard fiber that can distinguish the direction of bend. The sensing device was fabricated in two stages: the first stage involved LPG inscription and the second the introduction of an asymmetric change in the claddings refractive index. For the first stage, the LPG was inscribed into a single-mode standard communications step-index optical fiber with a core radius of $3.5 \mu \mathrm{m}$ and an outer cladding radius of $62.5 \mu \mathrm{m}$. The fiber was not specifically designed to be photosensitive and so its photosensitivity was increased by hydrogenation at a pressure of 120 Bar for a period of two weeks at room temperature. The LPG was fabricated using a frequency-doubled argon laser by sequential writing technique. The LPGs grating period was $400 \mathrm{~mm}$ and the length $5 \mathrm{~cm}$. The second stage involved modification of the cladding with a femtosecond laser system. The system differed from that described above by the addition of $f=100 \mathrm{~mm}$ cylindrical lens put in the proximity of the $\times 100(\mathrm{NA}=0.55)$ microscopic objective. The inclusion of the cylindrical lens modifies the spot size and shape of the laser beam at the point of inscription. Three continuous waveguides (refractive index change of about $1.5 \times 10^{-3}$ ) were written in the same plane and parallel to the core. Two of the waveguides were placed $2 \mu \mathrm{m}$ either side of the core and the third waveguide was placed $20 \mu \mathrm{m}$ from the core. The intensity of the laser was kept at a level to make single spots overlap, thus making the waveguides continuous. The diameter of each waveguide was approximately $5 \mu \mathrm{m}$. The LPG device was inserted into a $4 \mathrm{~mm}$ diameter flexible rubber tube and both the fiber and the rubber tube were clamped between two towers with one of the clamps mounted on a translation stage. This was moved inwards to induce a bend in the rubber tube and hence the optical fiber. We describe the bending as convex when the waveguide written $20 \mu \mathrm{m}$ from the core is on the outermost side of the bend, and concave when this waveguide is on the inside of the bend. The tube helped ensure that the fiber does not flip to another orientation. For this arrangement, where the LPG is midway between the clamps, the sensors curvature, $\mathrm{R}$, is given by ${ }^{17}$

$$
R=\frac{2 d}{d^{2}+L^{2}}
$$


where $L$ is the half distance between the edges of the two towers and $d$ is the bending displacement at the centre of the LPG. The experimental characterization of the LPGs attenuation bands was carried out by illuminating the device using a broadband light source and observing the transmission spectrum with an optical spectrum analyzer with an accuracy of $0.05 \mathrm{~nm}$. The results are shown in Fig. 8 for concave and convex bending.

First, the spectral sensitivity of the normal attenuation bands (those present in the absence of any bending) was measured for both modes of bending, resulting in $d \lambda / d R=+5.6 \pm 0.1 \mathrm{~nm} / \mathrm{m}$ for concave bending and $d \lambda / d R=+4.6 \pm 0.2 \mathrm{~nm} / \mathrm{m}$ for convex bending. Secondly the bend induced attenuation band was characterised and displayed a spectral sensitivity of $d \lambda / d R=+1.6 \pm 0.1 \mathrm{~nm} / \mathrm{m}$ for concave bending and $d \lambda / d R=+3.8 \pm$ $0.5 \mathrm{~nm} / \mathrm{m}$ for convex bending. The wavelength shifts as a function of curvatures are shown in Fig. 2. This spectral behavior is explained by the introduction of the asymmetry as seen in the response to bending of LPGs in D-shaped fiber. ${ }^{15}$ This LPG device was modeled using a finite-element package (Femlab) to obtain the effective refractive indices of the core and the cladding modes at various curvatures (concave and convex bending) at a given wavelength. The presence of bending was treated by using the conformal mapping technique, ${ }^{18}$ which replaces the curved optical fiber waveguide by an equivalent straight waveguide with the following index profile as a function of curvature $R$ and longitudinal strain $\epsilon$ :

$$
n(R)=\left(n(0, \lambda)+\frac{d n}{d \epsilon}\right) \exp \left(\frac{d}{R}\right)
$$

where $n(0)$ is the initial refractive index contrast of the core and cladding at a wavelength of $\lambda, R$ is the curvature experienced by the fiber and $d$ is the distance from the center of the fiber. The modeling produced the same general spectral behavior with respect to curvature, but the spectral sensitivities of the various attenuation bands were different; this is not surprising as the exact refractive index profile of the LPG device is not known.

\subsection{All-femtosecond made LPG}

Since the early demonstrations of grating inscription in various fibers using a femtosecond laser ${ }^{6}$ much effort has been spent to improve the quality of fiber gratings and to understand the mechanisms of the residual changes of the refractive index. ${ }^{19}$ It was previously reported that a femtosecond post-processing of LPG fabricated by means of standard UV inscription results in a new type of fiber device - a directional bend sensor. ${ }^{20}$ In this section we demonstrate all-femtosecond technology for fabrication of LPG-based polarization sensitive fiber devices in a standard telecommunication fiber without prior photosensitization. In our approach, we have used a sharp focusing x100 microscopic objective as opposed to typically used long focal distance optics. This allows for accurate positioning of the small modified area with respect to the fiber axis. Hence the controlled asymmetry is introduced in the resulting device. Further advantage is that the grating inscription and its post-processing are now combined in a single fabrication stage. Finally, the spectral stability against thermal annealing or under elevated temperatures was found to be much higher. ${ }^{11}$ We have also found that asymmetrically fabricated LPGs have better sensitivity to bending at lower curvature $\left(0.3 \mathrm{~m}^{-1}\right)$ as compared to a standard UV-LPG in the same fiber. This suggests high polarization sensitivity of such gratings which is the main focus of this experiment.

The standard SMF-28 fiber was mounted on the computer controlled 2D-stage and moved with a typical speed of $0.07 \mathrm{~mm} / \mathrm{s}$. Variation of the electrical pulse period and the fiber translation speed enabled fabrication of a series of LPGs with periods between 0.33 and $0.35 \mathrm{~mm}$. For the parameters mentioned above, it takes 5 seconds to inscribe one period (pitch) of the LPG. The inscription of the grating was done after the careful positioning of the focal spot with respect to the fiber core on both ends of the scanning range with the two auxiliary 3Dstages (5). We used a long working distance $\times 100$ objective. A visible groove was typically produced inside the core of the fiber rather than a smooth track. A series of gratings with periods around $0.35 \mathrm{~mm}$ and a length of $45 \pm 5 \mathrm{~mm}$ have been made for the range of pulse energies. After the inscription, the microscopic images (Fig. 9) of all gratings were captured to check their positions in relation to the fiber axis. All gratings were offset by approximately the same distance from the centre of the core, as illustrated by Fig. 9. The transmission spectrum of one of the LPGs made by the femtosecond inscription is shown in Fig. 10. A typical spectrum of the standard UV inscribed LPG is shown in the insert for comparison. The transmission spectrum of the gratings is characterized by means of an optical spectrum analyzer with a $0.1 \mathrm{~nm}$ resolution. The light from the broadband source was launched into the grating under test after passing through the polarizer and a polarization 


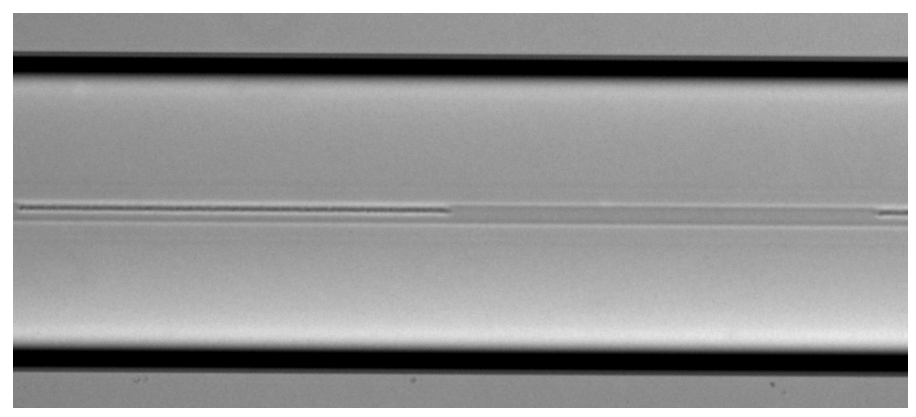

Figure 9. Microscopic image of the fiber with LPG. The groves are offset with respect to the core axis.

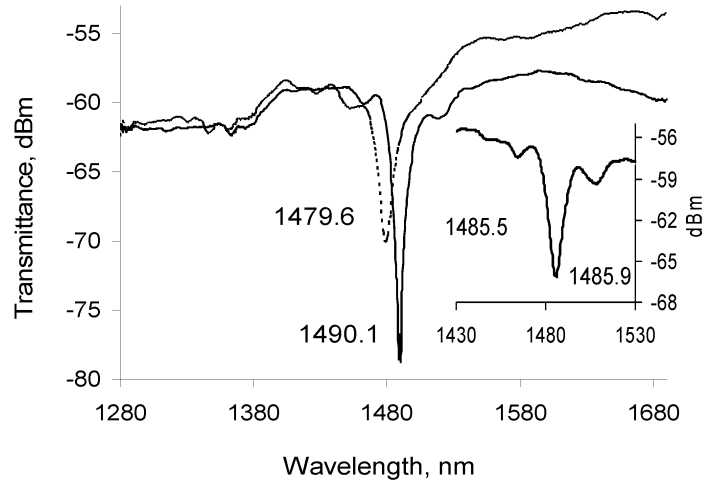

Figure 10. The transmission spectrum of the LPG made by the femtosecond laser. The positions of dips are marked on the graph. The insert shows the spectrum of the standard LPG made by a UV laser after hydrogenation.

controller. Figure 10 suggests, that the strength of the attenuation band for the long period grating written by the femtosecond laser is approximately $20 \mathrm{~dB}$, which is comparable to the UV inscribed LPG. Secondly, the LPG produced with femtosecond laser has a very strong dependence in the transmission dip positions for different polarizations $(10.5 \mathrm{~nm})$, compared with the standard UV-written LPG $(0.4 \mathrm{~nm})$. Another distinctive feature was observed while measuring the polarization splitting versus the pulse energy used for inscription. It is clear that fs-inscribed-LPG tends to be more "birefringent" when higher pulse energy is used. The latter can be attributed to the stress induced by the femtosecond laser in the material, surrounding the modified area. ${ }^{19}$ The visible size of the fabricated structure is less than the area affected by the laser, which could explain a big overlapping integral and strong attenuation bands. This was also confirmed by the thermal annealing results. Alternative explanation is that the perturbation of the waveguide, done by the femtosecond laser is not small, and the modal structure of the resulting waveguide is significantly different to that of the original fiber. Numerical simulations confirm a strong birefringence (the dependence of the effective refractive indexes for two orthogonal polarizations) even for a couple of fundamental modes.

\section{THERMAL STABILITY OF FS-INSCRIBED FIBER BRAGG GRATINGS}

Growing interest in femtosecond laser inscription is based, among other factors, on the fact that the physical mechanism of this process is essentially different to that of UV inscription. As a result, the physical properties of the fs-inscribed structures and devices are different to those of the UV-produced equivalents. The refractive index change in fs-inscribed structures is believed to be initiated by formation of localized plasma in the bulk of the material causing densification of the latter. The process involves highly nonlinear photo-ionization, thus requiring high intensity of light and it only occurs in a tightly focused laser beam. Previous reports indicate that, contrary to UV inscription, fs-inscription does not depend strongly on formation of defects. ${ }^{21}$ As a result, the 


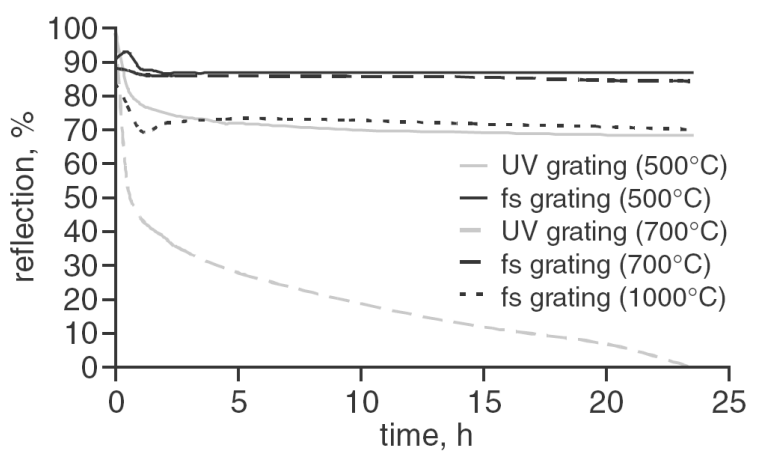

a

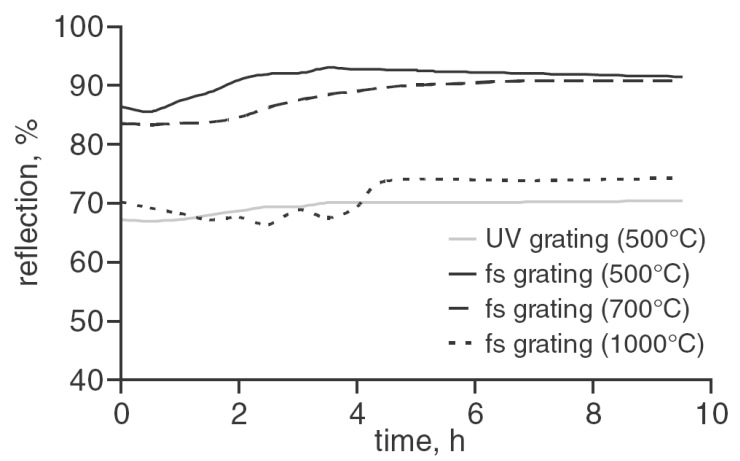

$\mathrm{b}$

Figure 11. Isothermal evolution of reflection of fs-inscribed and UV-inscribed gratings during $24 \mathrm{~h}$ annealing and $10 \mathrm{~h}$ cooling down. (a) $24 \mathrm{~h}$ annealing (b) $10 \mathrm{~h}$ cooling.

decay of defects caused by thermal annealing is likely to be insignificant in the fs-inscribed structures. Hence, fs-inscription should produce structures with improved thermal robustness compared to UV-written structures. High thermal stability of the fs-inscribed structures has been observed recently in the FBGs produced by using a special, high-order phase mask. ${ }^{4}$ In this section, we present a study of thermal properties of FBGs produced using direct, point-by-point inscription. These devices present smaller modified areas and higher refractive index contrast than gratings inscribed with a femtosecond laser and phase mask. We established that these FBGs are stable up to a temperature of $900^{\circ} \mathrm{C}$. Annealing at temperatures up to $700^{\circ} \mathrm{C}$ actually increased the grating reflectivity, indicating the relaxation of strains induced during inscription in the vicinity of the modified region. Experimental setup and inscription procedure were similar to those described above. The beam was focused into the fiber core by a $\times 100$ microscopic objective. Standard fiber (SMF) was used; no photosensitization procedure was carried out prior to the exposure.

Pulse energy was set to approximately $0.5 \mathrm{~mJ}$ corresponding to the peak intensities in the order of $10^{14}$ $\mathrm{W} / \mathrm{cm}^{2}$. The gratings were approximately $30 \mathrm{~mm}$ long. The pitch size in the gratings used in this work was $1.07 \mu$, corresponding to the second order of the Bragg reflection at a wavelength of $1.55 \mu$. Typical spectral characteristics of a fs-inscribed FBG are presented in Fig. 2, and are consistent with those reported previously. ${ }^{6}$ Three similar samples of the fs-inscribed FBGs with reflectivity ranging from $80 \%$ to $90 \%$ were placed in an oven and annealed at constant temperatures of 500,700 and $1000^{\circ} \mathrm{C}$, respectively, for a period of 24 hours. The grating spectra were monitored every $30 \mathrm{~min}$ by an analyzer with a resolution of $5 \mathrm{pm}$. After the annealing period, the oven was switched off and the gratings were allowed to cool down to room temperature. Monitoring of the grating spectra with the $30 \mathrm{~min}$ intervals was continued during the first $10 \mathrm{~h}$ of the cooling process. Three UV-inscribed FBGs were used as control samples. These were inscribed in hydrogenated fiber by using a $90 \mathrm{~mW}$ beam from a CW laser operating at a wavelength of $244 \mathrm{~nm}$. After inscription, the FBGs were post-processed by annealing at $80^{\circ} \mathrm{C}$ for 24 hours. The resultant reflectivity of control samples before the tests was in excess of $98 \%$. The three control samples were subjected to the procedures of annealing and measurement identical to the ones applied to the fs-inscribed samples as described above.

Measured evolution of the grating reflection is presented in Fig. 11. Femtosecond inscribed gratings showed a significantly improved thermal stability compared to the hydrogenated, UV-inscribed FBGs. The UV-inscribed sample experienced a significant degradation at $500^{\circ} \mathrm{C}$ and was rapidly erased at $700^{\circ} \mathrm{C}$, whilst the fs-inscribed FBG did not show any signs of rapid decay up to $1000^{\circ} \mathrm{C}$. Comparison with similar studies from the literature shows that the thermal stability of the fs-inscribed FBGs is better than that of common type I and type IIA gratings and is similar to the stability of the type II gratings, based on optical damage. ${ }^{22}$

In all cases, we observed that the grating reflectivity after cooling down to room temperature was greater than that at the corresponding high temperature. This could be explained by relaxation of mechanical stress created in the outer regions of the modified area. Similar annealing behavior has been previously observed in fs-inscribed photonic crystals. ${ }^{23}$ At lower temperature levels of 500 and $700^{\circ} \mathrm{C}$, this effect dominated in 


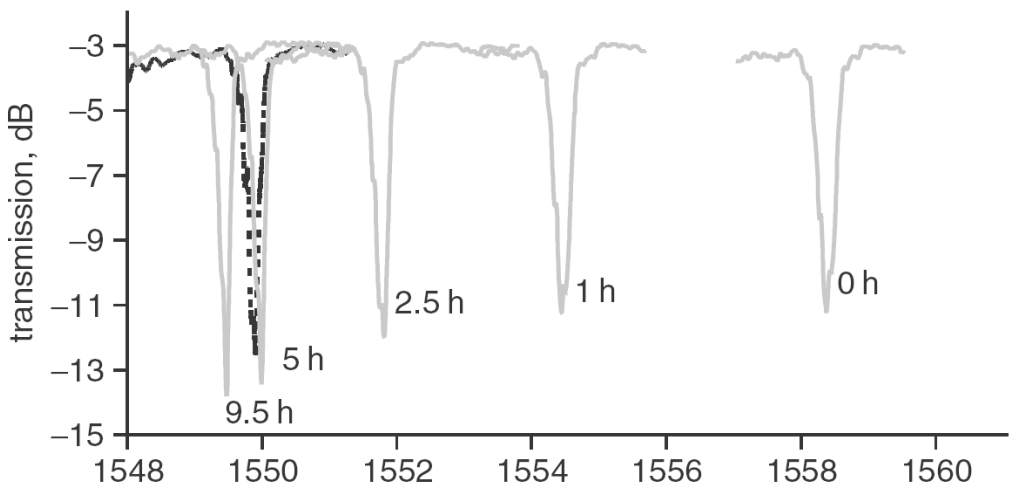

Figure 12. Spectral evolution of FBG during cooling down period after annealing at $700^{\circ} \mathrm{C}$ (gray lines). Dashed line shows the original spectrum before annealing.

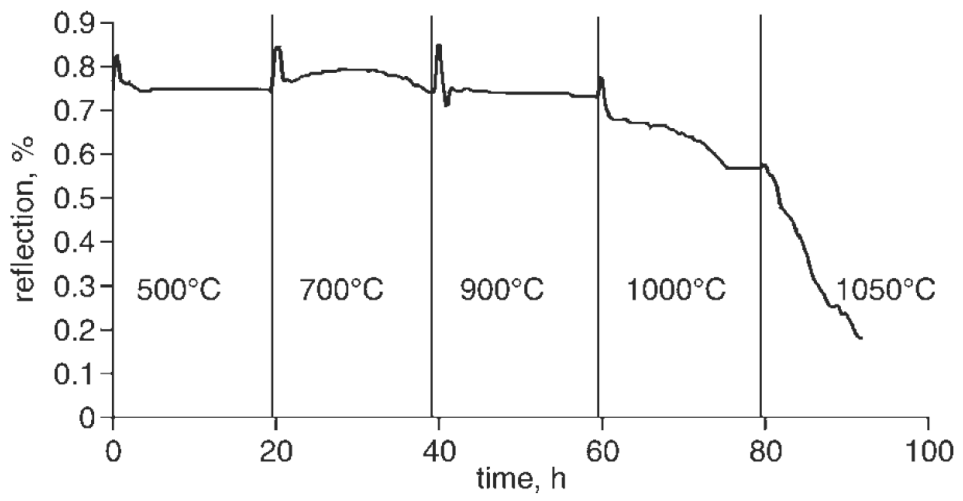

Figure 13. Dynamics of reflection in fs-inscribed FBG during annealing at increasing temperatures.

fs-inscribed gratings and the resulting reflectivity actually increased after the annealing-cooling cycle in those gratings. Fig. 12 shows the dynamics of grating spectra during the coolingdown period. The measurements were taken after annealing for $24 \mathrm{~h}$ at $700^{\circ} \mathrm{C}$. The measured spectral shift of $12.8 \mathrm{pm} /{ }^{\circ} \mathrm{C}$ is in line with the values of approximately $13 \mathrm{pm} /{ }^{\circ} \mathrm{C}$, reported earlier for the UV-written FBGs. ${ }^{24}$

In the next experiment, the same fs-inscribed grating was annealed successively at increasing temperatures of $500,700,900,1000$ and $1050^{\circ} \mathrm{C}$. Annealing at each temperature lasted for 20 hours, after which the grating was allowed to cool down for 5 hours to room temperature before the next annealing cycle. The dynamics of the grating reflection during this exercise is shown in Fig. 13. First, the grating reflection dropped during each heating period and subsequently increased during the cooling period, similar to the behavior during the previous experiment, described above. The reflectivity decrease caused by annealing at lower temperatures was reversible. A certain increase in reflectivity was observed after the heating-cooling cycles at temperatures of 500 and $700^{\circ} \mathrm{C}$. The $900^{\circ} \mathrm{C}$ cycle produced a slight overall decrease of reflectivity, and the $1000^{\circ} \mathrm{C}$ cycle caused a significant permanent degradation of the grating performance. Finally, annealing at a temperature of $1050^{\circ} \mathrm{C}$ practically erased the grating, rapidly and irreversibly reducing the reflection coefficient to a level below $20 \%$. Overall, the results in this work are complementary to those reported in previous studies of the thermal behavior of the structures inscribed in glass by ultrafast lasers. ${ }^{4}$ No significant difference was established between the observed annealing behavior of the structures written directly by a tightly focused ultrafast laser beam and the reported earlier high temperature tests of the gratings, produced with a phase mask by a defocused beam of a similar laser. 


\section{CONCLUSIONS}

Direct femtosecond microfabrication, reviewed in this paper requires neither phase-masks nor photosensitized fibers and hence offers remarkable technological flexibility. Unlike known methods of making FBGs and LPGs, very short inscription time is required. High quality FBGs and LPGs were produced using this method in commercial, non-photosensitized, unhydrogenated fibers. We have also fabricated radially asymmetric and more complicated 3D structures. Such structures demonstrate unprecedented sensitivity to directional bending and polarization properties. The prototypes of FBG- and LPG-based vector bending sensors have been developed. Thermal properties of the above described structures fs-fabricated were examined. Gratings were thermally stable at temperatures up to $900^{\circ} \mathrm{C}$, representing a significant improvement in comparison with the conventional UV-inscribed gratings.

\section{ACKNOWLEDGMENTS}

The support of DTI in the framework of LASEWAVE project is acknowledged.

\section{REFERENCES}

1. Y. Kondo, K. Nouchi, T. Mitsuyu, M. Watanabe, P. G. Kazansky, and K. Hirao, "Fabrication of long-period fiber gratings by focused irradiation of infrared femtosecond laser pulses," Opt. Lett. 24, p. 646648, 1999.

2. E. Fertein, C. Przygodzki, H. Delbarre, A. Hidayat, M. Douay, and P. Niay, "Refractive-index changes of standard telecommunication fiber through exposure to femtosecond laser pulses at 810 nm," Appl. Opt. 40, pp. 3506-3508, 2001.

3. S. J. Mihailov, C. W. Smelser, D. Grobnic, R. B. Walker, P. Lu, H. Ding, and J. Unruh, "Bragg gratings written in all- $\mathrm{sio}_{2}$ and ge-doped core fibres with 800-nm femtosecond radiation and a phase mask," $J$. Lightwave Technol. 22, pp. 94-100, 2004.

4. D. Grobnic, C. W. Smelser, and S. J. Mihailov, "Isothermal behavior of fibre bragg gratings made with ultrafast laser at temperatures above 1000oc," ECOC 2004 ECOC 2004 Proc., p. Paper Tu1.3.5, 2004.

5. A. Dragomir, K. A. Z. P. G. Kryukov, E. M. Dianov, and D. N. Nikogosyan, "Inscription of fibre bragg gratings by ultraviolet femtosecond radiation," Opt. Lett. 28, pp. 2771-2173, 2003.

6. A. Martinez, M. Dubov, I. Khrushchev, and I. Bennion, "Direct writing of fibre bragg gratings by femtosecond laser," Electron. Lett. 40, pp. 1170-1172, 2004.

7. B. Malo, K. O. Hill, F. Bilodeau, D. C. Johnson, and J. Albert, "Point-by-point fabrication of microbragg gratings in photosensitive fibre using single excimer pulse refractive index modification techniques," Electron. Lett. 29, pp. 1668-1669, 1993.

8. P. P. Pronko, S. K. Dutta, J. Squier, J. V. Rudd, D. Du, and G. Mourou, "Machining of sub-micron holes using a femtosecond laser at 800 nm," Opt. Commun. 114, pp. 106-110, 1995.

9. F. M. Araujo, L. A. Ferreira, J. L. Santos, F., and Farahi, "Temperature and strain insensitive bending measurements with d-type fibre bragg gratings," Meas. Sci. Technol. 12, pp. 829-833, 2001.

10. D. Zhao, L. Zhang, and I. Bennion, "Implementation of vector bending sensors using long-period gratings uv-inscribed in flat-clad and 4-core fibres," Int. Conf. on Optical Fibre Sensors, Nara, Japan, pp. Paper OFS-16, 2003.

11. A. Martinez, I. Khrushchev, and I. Bennion, "Thermal properties of fibre bragg gratings inscribed pointby-point by infrared femtosecond laser," Electron.Lett. 41, pp. 176-178, 2005.

12. T. Erdogan, "Cladding-mode resonances in short- and long-period fibre grating filters," J. Opt. Soc. Am. A 4, pp. 1760-1773, 1997.

13. A. Kersey, M. Davis, H. Patrick, M. LeBlanc, K. P. Koo, C. Askins, M. Putnam, and E. Frieble, "Fibre grating sensors," J. Lightwave Technol. 15, pp. 1442-1463, 1997.

14. T. Allsop, T. Earthrowl-Gould, D. J. Webb, and I. Bennion, "Embedded progressive-three-layered fiber long-period gratings for respiratory monitoring," SPIE J. Biomed. Opt. 8, pp. 552-558, 2003.

15. T. Allsop, A. Gillooly, V. K. Mezentsev, T. Earthgrowl-Gould, R. Neal, D. J. Webb, and I. Bennion, "The spectral characteristics of long period gratings written in d-shaped optical fiber as bending sensors," OFS '2003 16th International Conference on Optical Fibre Sensors, pp. Paper Tu1.3.5 pp. 88-91, 2003. 
16. H. Patrick, "Self-aligning, bipolar bend transducer based on long period grating written in eccentric core fibre," Electron. Lett. 36, pp. 21-22, 2000.

17. W. Du, H. Tam, M. Liu, and X. Tao, "Long-period grating bending sensors in laminated composite structures," SPIE Conf. Proc. Smart structures and materials 3330, p. 284, 1998.

18. M. Heiblum and J. Harris, "Analysis of curved optical waveguides by conformal transformation," Quantum Electron. QE-11, pp. 75-83, 1975.

19. F. Durr, H. G. Limberger, R. P. Salathe', F. Hindle, M. Douay, E. Fertein, and C. Przygodzki, "Tomographic measurement of femtosecond-laser induced stress changes in optical fibers," Appl.Phys.Lett. 84, pp. 49834985, 2004.

20. T. Allsop, M.Dubov, A. Martinez, F. Floreani, I. Khrushchev, D.J.Webb, and I. Bennion, "Long period grating directional bend sensor based upon an asymmetric index modification of cladding," Electron. Lett. 41, pp. 59-60, 2005.

21. A. M. Streltsov and N. F. Borrelli, "Study of femtosecond laser written waveguides in glasses," JOSA B 19, pp. 2496-2504, 2002.

22. A. Othonos and K. Kalli, Fibre Bragg gratings: fundamentals and applications in telecommunications and sensors Guide to Technical Editing, Artech House, 1999.

23. H. B. Sun, Y. Xu, S. Juodkazis, K. Sun, M. Watanabe, S. Matsuo, H. Misawa, and J. Nishii, "Arbitrarylattice photonic crystals created by multiphoton microfabrication," Opt. Lett. 26, pp. 325-327, 2001.

24. B. T. Kersey, A.D. and W. Morey, "High-resolution fibre grating based strain sensor with interferometric wavelength shift detection," Electron. Lett. 28, pp. 236-238, 1992. 